\title{
Correction to: Establishment and validation of a method for cell irradiation in 96-well and 6-well plates using a linear accelerator
}

\author{
Xiao-Qing Dong ${ }^{1} \cdot$ Qing Lin $^{1} \cdot$ Jie Hu${ }^{1} \cdot$ Liang Huang $^{2} \cdot$ Kun Yue $^{3} \cdot$ \\ Lu Wang ${ }^{1} \cdot$ Jia-Fei Zhang ${ }^{1} \cdot$ Mei-Ling Lu ${ }^{4}$
}

Published online: 11 April 2018

(C) Shanghai Institute of Applied Physics, Chinese Academy of Sciences, Chinese Nuclear Society, Science Press China and Springer Nature Singapore Pte Ltd. 2018

Correction to: NUCL SCI TECH (2018)29:67

https://doi.org/10.1007/s41365-018-0407-z

In the original publication, Figure $2 \mathrm{c}$ is incorrectly published. The correct figure is provided in this correction article.

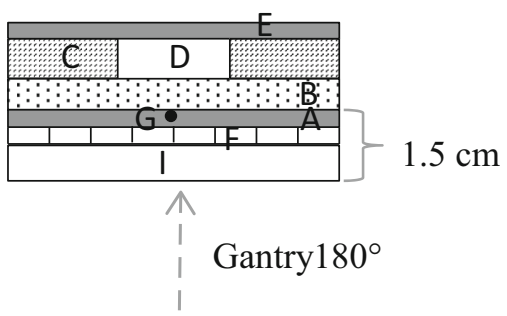

The original article can be found online at https://doi.org/10.1007/ s41365-018-0407-z.

Qing Lin

linqing.linda@163.com

Xiao-Qing Dong

dxq_xiaoqing@163.com

1 Department of Radiation Oncology, Tenth People's Hospital Affiliated to Tongji University, Shanghai 200072, China

2 Medical Equipment Department, Tenth People's Hospital Affiliated to Tongji University, Shanghai 200072, China

3 Department of Radiation Oncology, (North) NO.9 People's Hospital affiliated to Shanghai Jiao Tong University School of Medicine, Shanghai 201999, China

4 Central Lab, Tenth People's Hospital Affiliated to Tongji University, Shanghai 200072, China 